\title{
Using Indirect Covariance Spectra to Identify Artifact Responses in Unsymmetrical Indirect Covariance Calculated Spectra
}

\author{
Gary E. Martin* and Bruce D. Hilton \\ Schering-Plough Research Institute \\ Rapid Structure Characterization Laboratory \\ Pharmaceutical Sciences \\ Summit, NJ 07901
}

Kirill A. Blinov

Advanced Chemistry Development

Moscow Division

Moscow 117504

Russian Federation

and

Antony J. Williams

ChemZoo, Inc.

Wake Forest, NC 27581

\footnotetext{
* To whom inquiries should be addressed Schering Plough Research Institute Rapid Structure Characterization Laboratory Mail Stop S7-D1

556 Morris Ave

Summit, NJ 07901

(908) 473-5398

(908) 473-6559 (fax)

gary.martin@spcorp.com
} 


\begin{abstract}
Several groups of authors have reported studies in the areas of indirect and unsymmetrical indirect covariance NMR processing methods. Efforts have recently focused on the use of unsymmetrical indirect covariance processing methods to combine various discrete two-dimensional NMR spectra to afford the equivalent of the much less sensitive hyphenated 2D NMR experiments, for example GHSQC-COSY and GHSQCNOESY. Alternatively, unsymmetrical indirect covariance processing methods can be used to combine multiple heteronuclear 2D spectra to afford ${ }^{13} \mathrm{C}-{ }^{15} \mathrm{~N}$ HSQC-HMBC correlation spectra. Now we wish to report the use of responses contained in indirect covariance processed GHSQC spectra as a means for the identification of artifacts in both indirect covariance and unsymmetrical indirect covariance processed 2D NMR spectra.
\end{abstract}




\section{INTRODUCTION}

The first reported application of indirect covariance processing of heteronuclear 2D NMR data was in the late 2004 report of Zhang and Brüschweiler who applied the method to a GHSQC-TOCSY spectrum to produce a ${ }^{13} \mathrm{C}-{ }^{13} \mathrm{C}$ connectivity plot. ${ }^{1} \mathrm{~A}$ footnote in that 2004 report mentioned the possibility of proton resonance overlap causing artifacts in the ${ }^{13} \mathrm{C}-{ }^{13} \mathrm{C}$ connectivity plot but the authors did not elaborate further on that observation. Using several model compounds, in 2005 the present authors reported the analysis of two types of artifacts in ${ }^{13} \mathrm{C}-{ }^{13} \mathrm{C}$ connectivity plots derived from indirect covariance processing of IDR-GHSQC-TOCSY spectra (Inverted Direct Response-GHSQC-TOCSY). ${ }^{2}$ In an effort to eliminate artifact responses, the indirect covariance processing algorithm was modified to allow two 2D NMR data matrices to be co-processed. By decomposing an IDR-GHSQC-TOCSY spectrum into the corresponding positively and negatively phased sub-spectra and then processing them back together using the unsymmetrical indirect covariance processing algorithm one type of artifact was eliminated and the other was rendered diagonally asymmetric, thereby allowing the second type of artifact response to be eliminated by symmetrization of the ${ }^{13} \mathrm{C}-{ }^{13} \mathrm{C}$ connectivity plot.

While the elimination of artifacts from ${ }^{13} \mathrm{C}-{ }^{13} \mathrm{C}$ connectivity plots is a useful attribute of the unsymmetrical indirect covariance processing algorithm, a far more useful capability resides in an investigator's ability to use the algorithm to co-process discretely acquired 2D NMR spectra. Examples have included: co-processing ${ }^{1} \mathrm{H}-{ }^{13} \mathrm{C}$ GHSQC and GHMBC spectra to yield the equivalent of an $m, n$-ADEQUATE spectrum ${ }^{3}$; coprocessing GHSQC and GCOSY spectra to yield GHSQC-COSY spectra ${ }^{4,5}$; co- 
processing GHSQC and NOESY data to produce the equivalent of GHSQC-NOESY spectra ${ }^{6}$; and co-processing ${ }^{1} \mathrm{H}_{-}{ }^{13} \mathrm{C}$ GHSQC and various ${ }^{1} \mathrm{H}_{-}{ }^{15} \mathrm{~N}$ long-range correlation spectra to derive ${ }^{13} \mathrm{C}-{ }^{15} \mathrm{~N}$ heteronuclear shift correlation spectra. ${ }^{7-9}$ It should be noted that the method is not restricted to gradient-based experiments with perhaps the sole exception of ${ }^{1} \mathrm{H}_{-}{ }^{15} \mathrm{~N}$ long-range correlation experiments, which cannot be reasonably performed without resorting to gradients.

\section{EXPERIMENTAL}

All data were acquired for the present study using a Varian $600 \mathrm{MHz}$ NMR spectrometer equipped with a $5 \mathrm{~mm}$ Cold Probe ${ }^{\mathrm{TM}}$ operating at an rf coil temperature of $20 \mathrm{~K}$. The sample used for the data acquisition was prepared by dissolving $\sim 1 \mathrm{mg}$ of naphtho[2', 1':5,6]naphtho [2',1':4,5] thieno[2,3-c]quinoline $(\mathbf{1})^{10}$ in $\sim 200 \mu \mathrm{L}$ deuterochloroform (CIL), after which the sample was transferred to a $3 \mathrm{~mm}$ NMR tube (Wilmad) using a flexible Teflon needle and a gas-tight syringe (Hamilton). Spectra acquired included a ${ }^{1} \mathrm{H}$ reference spectrum, a GCOSY spectrum (15 min), a GHSQC spectrum (75 min) and an $18 \mathrm{msec}$ IDR-GHSQC-TOCSY spectrum (6 hr). Experiments used the standard pulse sequences contained in the vendor-supplied pulse sequence library and were used without modification. Indirect and unsymmetrical indirect covariance processing was done using algorithms provided in ACD/Labs SpecManager v10.02 software. Data matrices were processed to afford identically digitized spectra $2 \mathrm{~K}$ $x 1 \mathrm{~K}$ points; spectral widths in $\mathrm{F}_{2}$ and $\mathrm{F}_{1}$ were not identical. Unsymmetrical indirect covariance processing times were typically a few seconds. 


\section{RESULTS AND DISCUSSION}

While examining the $\mathrm{icv}-{ }^{13} \mathrm{C}-{ }^{15} \mathrm{~N}$ HSQC-HMBC (icv =indirect covariance calculated; the notation HSQC-HMBC defines the heteronuclides involved, ${ }^{13} \mathrm{C}$ in the case of the HSQC portion of the acronym and ${ }^{15} \mathrm{~N}$ for the HMBC portion) spectrum of the anti-tumor alkaloid vinblastine, an artifact was observed due to the overlap of the $\mathrm{H} 2$ methine and 24-O-methyl singlets. ${ }^{11}$ The former was expected to exhibit a two-bond correlation to the N1 resonance; the correlation to the 24-O-methyl resonance, six bonds distant from N1, was clearly an artifact. Titrating the sample with $\mathrm{d}_{6}$-benzene demonstrated that Aromatic Solvent Induced Shifts (ASIS) ${ }^{12}$ could remove the degeneracy and eliminate the artifact response. Perhaps more importantly, it was also shown that the indirect covariance processed GHSQC spectrum (icv-HSQC hereafter) showed off-diagonal responses that predicted the location and identity of the O-methyl artifact response in the ${ }^{13} \mathrm{C}-{ }^{15} \mathrm{~N}$ icv-HSQC-HMBC spectrum. On that basis we wanted to explore the possibility of using off-diagonal responses in the icv-HSQC spectrum to identify artifact responses in icv- ${ }^{13} \mathrm{C}-{ }^{13} \mathrm{C}-\mathrm{COSY}$ correlation plots derived by indirect covariance processing of GHSQC-TOCSY spectra as well as artifact responses in icvHSQC-COSY/-TOCSY spectra derived by unsymmetrical indirect covariance coprocessing of gradient or non-gradient HSQC and either COSY or TOCSY spectra. As a model compound for the present study, we again elected to employ the complex polynuclear heteroaromatic, naphtho[ $\left.2^{\prime}, 1^{\prime}: 5,6\right]$ naphtho $\left[2^{\prime}, 1^{\prime}: 4,5\right]$ thieno[2,3-c] quinoline (1). ${ }^{10}$ The molecule has a congested ${ }^{1} \mathrm{H}$ NMR spectrum at $600 \mathrm{MHz}$ and multiple proton resonance overlaps that lead to artifact responses in the icv $-{ }^{13} \mathrm{C}-{ }^{13} \mathrm{C}$ COSY correlation plot derived from the IDR-GHSQC-TOCSY spectrum (see Figure 2 
for an explanation of the origins of the artifact responses) ${ }^{2}$ In a similar fashion, artifact responses are also anticipated in icv-HSQC-COSY spectra of $\mathbf{1}$ due to proton resonance overlap.

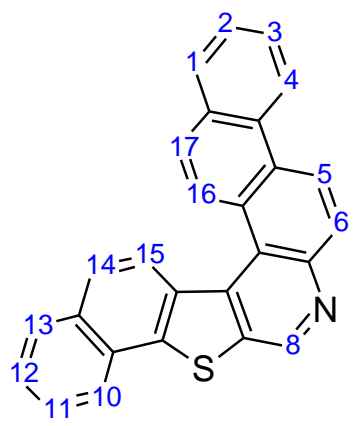

The GHSQC spectrum and the result obtained from the indirect covariance calculation are shown in Figures $1 \mathrm{~A}$ and B, respectively. As will be noted from the indirect covariance plot (icv-HSQC), there are pairs of off-diagonal responses observed due to proton resonance overlap of varying degrees that range from the essentially complete overlap of the $\mathrm{H} 6$ and $\mathrm{H} 15$ resonances to partial overlaps that give much weaker off-diagonal pairs in the icv-HSQC spectrum, as shown in Figure 1C. 


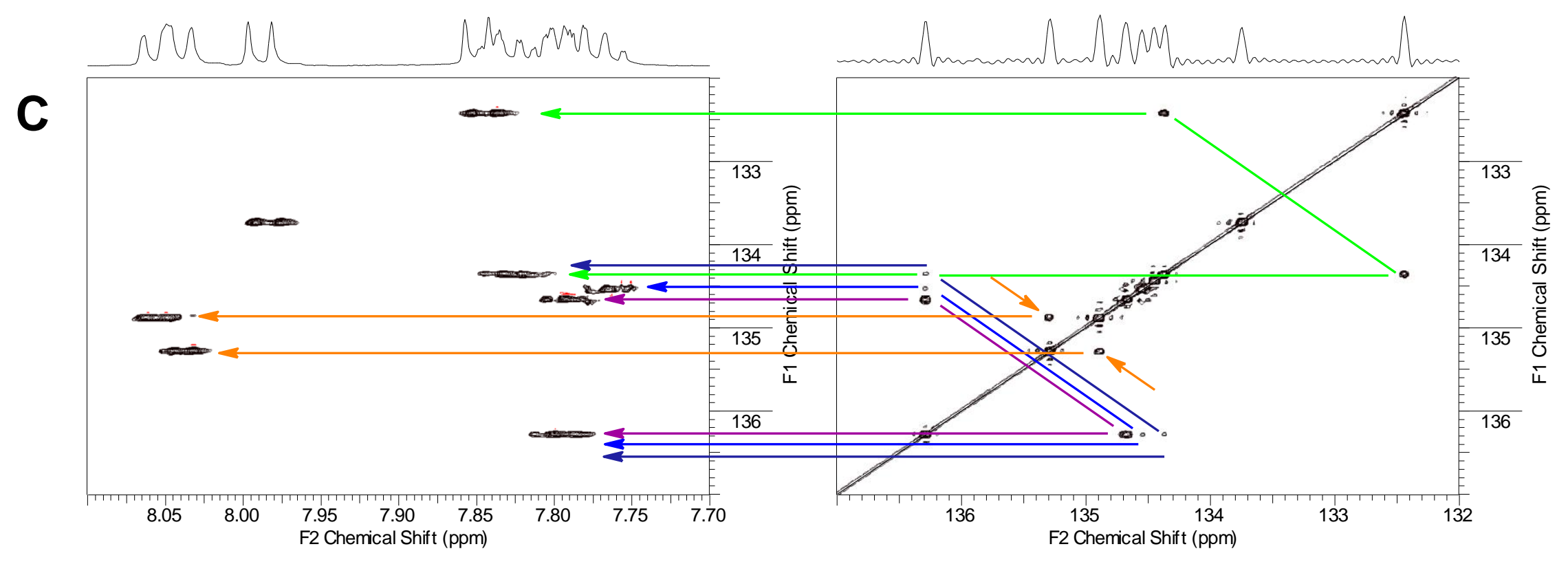

Figure 1. 


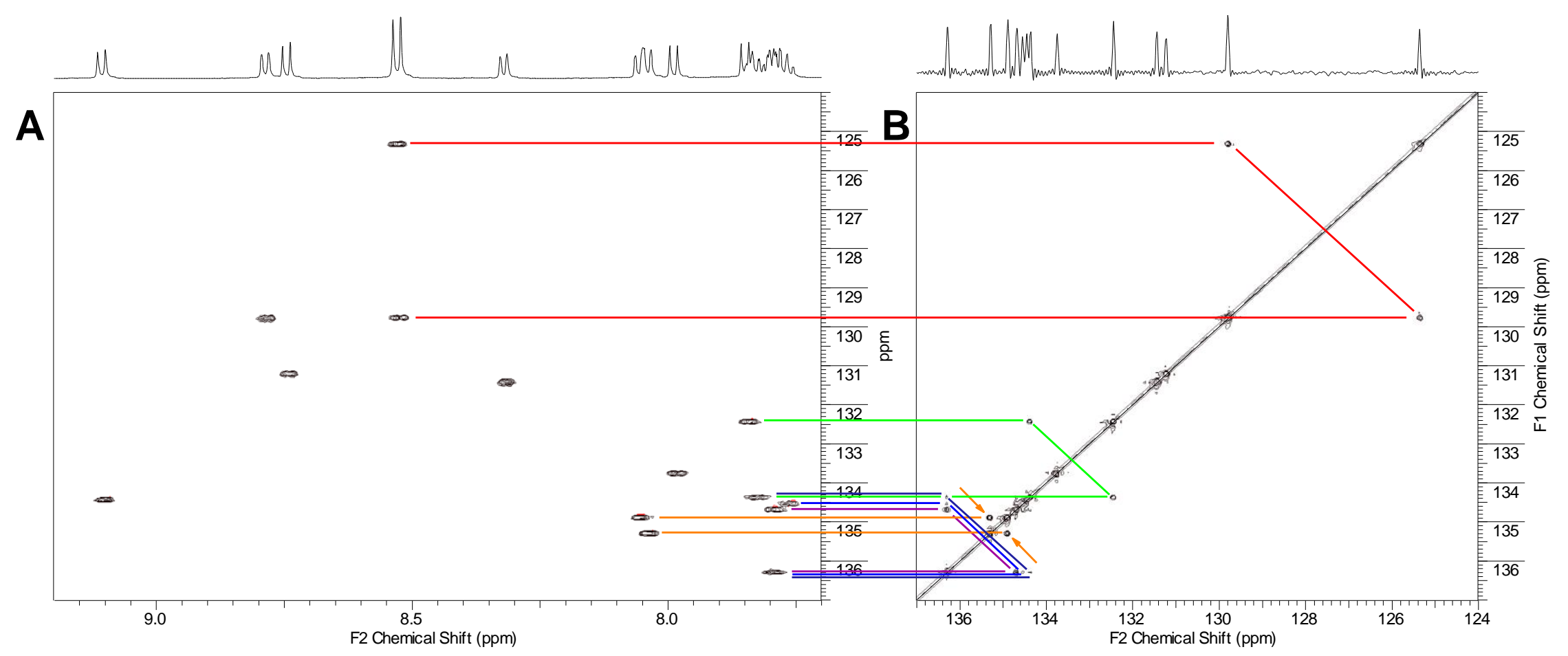

Figure 1. A) GHSQC spectrum of naphtho[2', ' $' 5,6$,6] naphtho[2', $\left.1^{\prime}: 4,5\right]$ thieno[2,3-c] quinoline (1) recorded at $25^{\circ} \mathrm{C}$ in $\mathrm{CDCl}_{3}$ at $600 \mathrm{MHz}$. B) Indirect covariance spectrum, icv-HSQC, calculated from the GHSQC spectrum shown in Panel A. Offdiagonal responses are observed only for overlapped proton resonances. C) An expansion of the region from 132-137 ppm plotted at a lower threshold level shows several of the weaker pairs of off-diagonal responses in the icv-HSQC spectrum that are barely visible in Panel B). As will be noted from Panel A, responses are generated in the indirect covariance processed spectrum shown in Panel B with overlap ranging from partial to full. 
Subjecting the IDR-GHSQC-TOCSY spectrum shown in Figure 2A to indirect covariance processing affords the icv- ${ }^{13} \mathrm{C}-{ }^{13} \mathrm{C}-\mathrm{COSY}$ connectivity plot shown in Figure 2B. Off-diagonal responses in Figure 2B correlated by solid black lines represent legitimate ${ }^{13} \mathrm{C}-{ }^{13} \mathrm{C}-\mathrm{COSY}$ correlation responses. Off-diagonal responses designated by solid red and dashed red lines correspond to Type I and Type II artifact responses, respectively. ${ }^{2}$ Figure 3 compares the indirect covariance spectrum calculated from the GHSQC spectrum shown in Panel A with the icv- ${ }^{13} \mathrm{C}-{ }^{13} \mathrm{C}-\mathrm{COSY}$ correlation plot calculated using indirect covariance processing shown in Panel B. Legitimate vicinal ${ }^{13} \mathrm{C}-{ }^{13} \mathrm{C}$-COSY correlations are denoted by solid black lines; Type I artifacts are designated by solid red lines; Type II artifacts are denoted by dashed black lines. The solid blue boxes linking Panels A and B identify the origins of the Type I artifact responses identified from the off-diagonal response in the icv-HSQC spectrum shown in Panel A. The single dashed blue box identifies the origins of one of the Type II artifact responses that arise from the overlap of a direct response from one spin system and a relayed response from a second, overlapped spin system.

While forecasting and identifying artifact responses in a icv- ${ }^{13} \mathrm{C}-{ }^{13} \mathrm{C}-\mathrm{COSY}$ correlation plot is interesting, being able to forecast the location of artifact responses in icv-HSQC-COSY spectra calculated using unsymmetrical indirect covariance processing is a much more useful application of the resonance overlap information data derived by icv-HSQC spectrum. Figure 4 shows the comparison of the unsymmetrical indirect covariance calculated icv-HSQC-COSY spectrum of $\mathbf{1}$ with the IDR-GHSQC-TOCSY spectrum recorded with an $18 \mathrm{~ms}$ mixing time shown previously in Figures 2A. In particular, the ability to use unsymmetrical indirect covariance processing methods to 

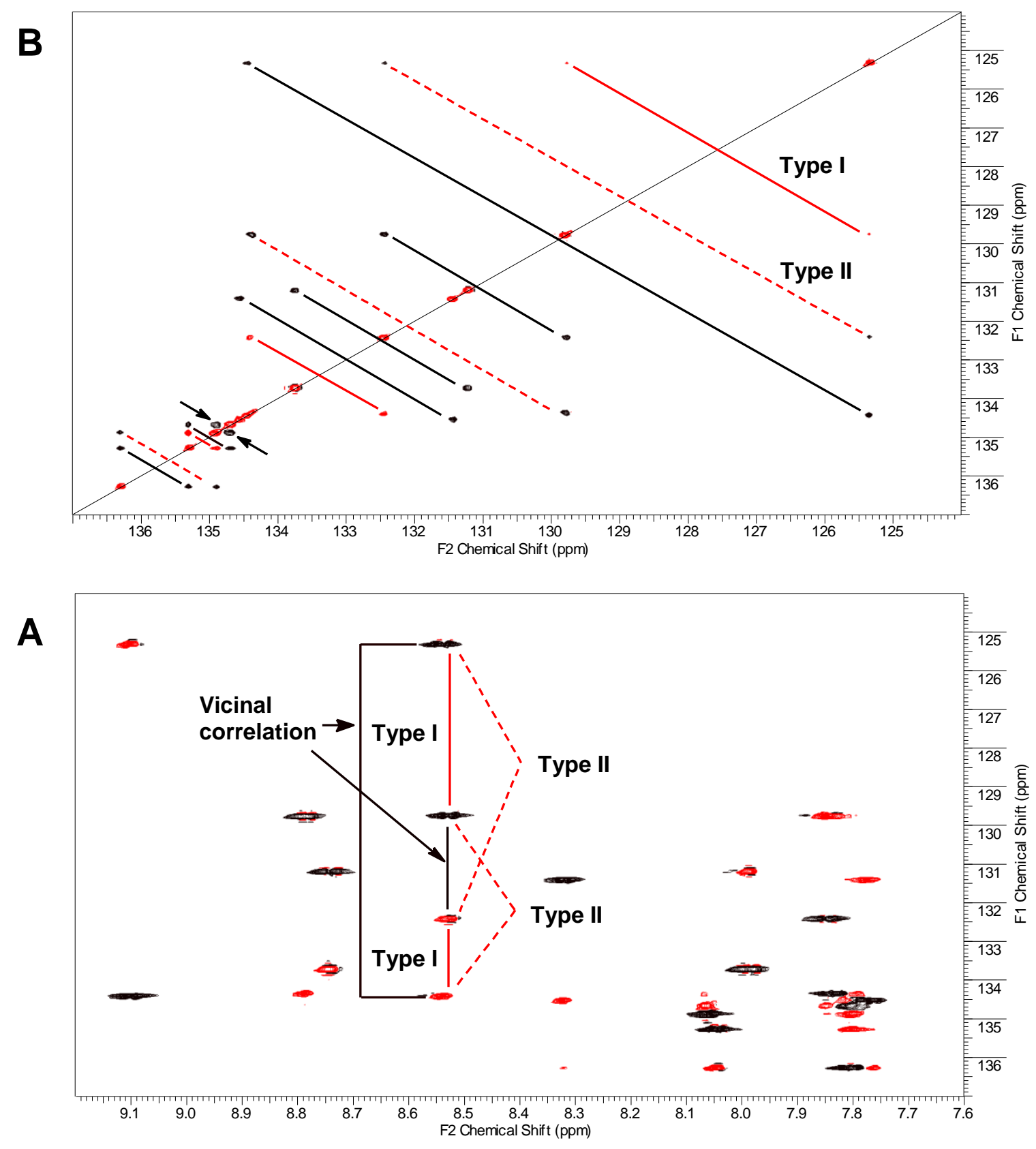

Figure 2. 
Figure 2. A.) The IDR-GHSQC-TOCSY spectrum of 1 recorded at $600 \mathrm{MHz}$ with an 18 msec mixing time. Direct responses are inverted and plotted in red; relayed responses have positive intensity and are plotted in black. Legitimate, vicinal correlations for the overlapped $\mathrm{H} 6$ and $\mathrm{H} 15$ protons are designated by solid black lines. Type I artifact responses arise between pairs of direct or relayed correlation responses and have negative phase in the indirect covariance processed spectrum shown in Panel B and are designated by solid red lines. Type II artifact responses arise between direct and relayed responses of different spin systems and have positive phase in the indirect covariance processed spectrum and are designated by dashed red lines in Panel B. Type I responses can be assigned by visual inspection; Type II responses can be assigned only through analysis of the spectrum. ${ }^{2}$ B.) The icv- ${ }^{13} \mathrm{C}-{ }^{13} \mathrm{C}$ correlation plot derived by the indirect covariance processing of the IDR-GHSQC-TOCSY spectrum shown in Panel A is presented in Panel B. Legitimate ${ }^{13} \mathrm{C}-{ }^{13} \mathrm{C}$ correlation responses are denoted by solid black lines. Type I artifact responses are shown by solid red lines; Type II artifact responses are denoted by dashed red lines. ${ }^{2}$ Additional artifact responses are observed when plots are prepared with a deeper threshold. 


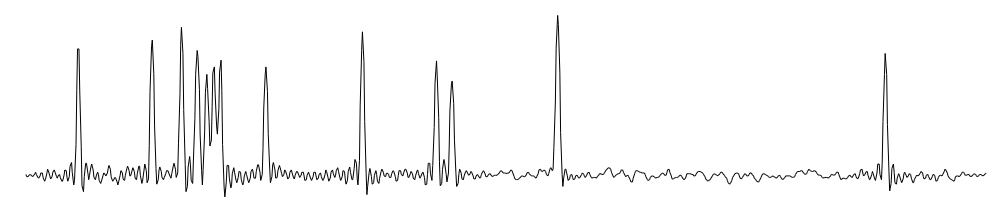

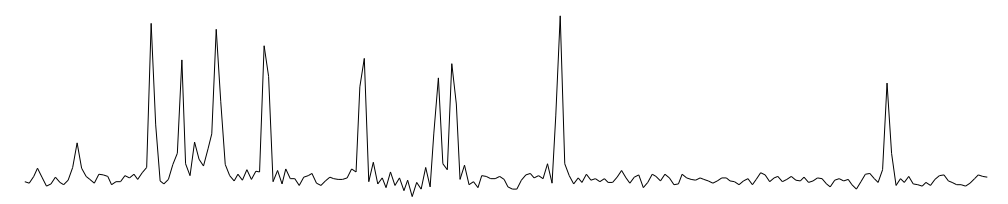

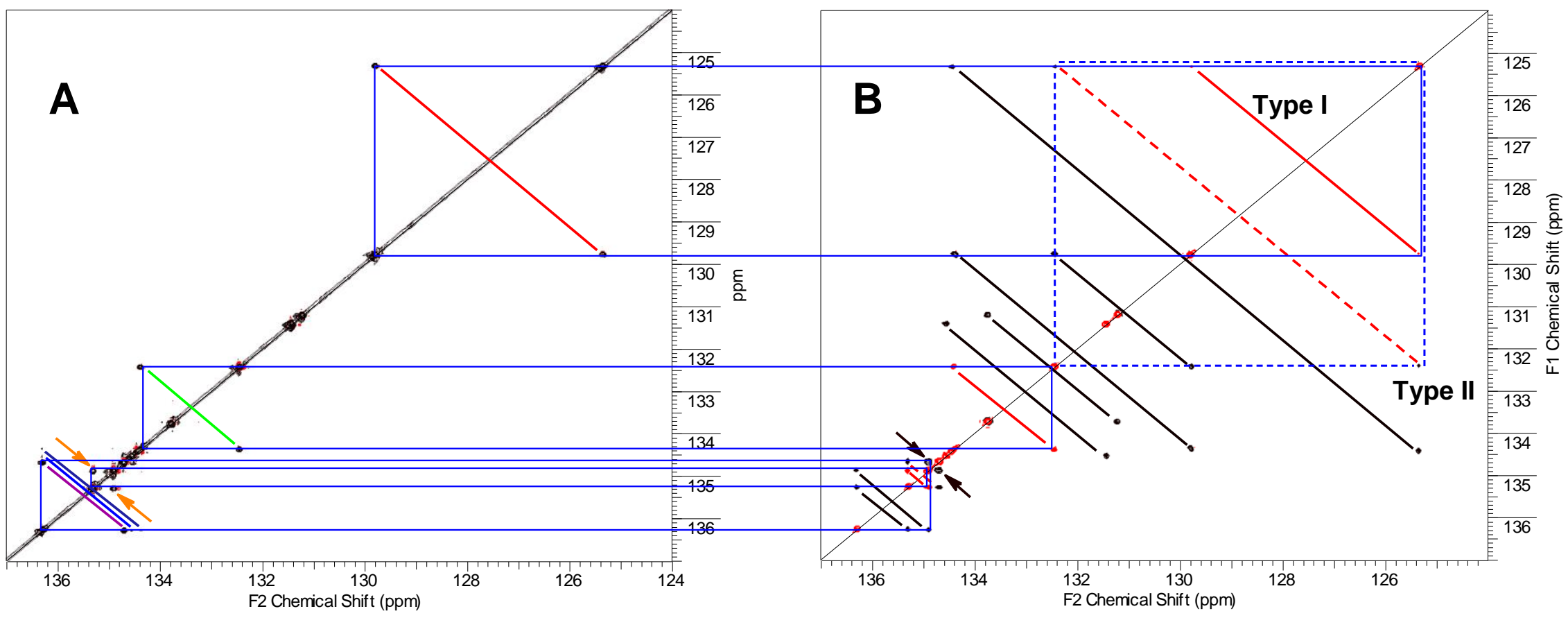

Figure 3. 
Figure 3. A.) Indirect covariance spectrum, icv-HSQC, calculated from the GHSQC spectrum of 1. Off-diagonal responses denote proton resonance overlaps that can give rise to artifact responses in the icv- ${ }^{13} \mathrm{C}-{ }^{13} \mathrm{C}-\mathrm{COSY}$ correlation plot prepared by indirect covariance processing of the GHSQC-TOCSY or IDR-GHSQC-TOCSY spectrum. Off-diagonal responses in panel A are color coded identically to Figure $1 \mathrm{~B}$ and C. B.) The icv- ${ }^{13} \mathrm{C}-{ }^{13} \mathrm{C}-\mathrm{COSY}$ plot prepared by the indirect covariance processing of the IDR-GHSQC-TOCSY spectrum shown in Figure 2A. Off-diagonal responses designated with solid black lines correspond to legitimate ${ }^{13} \mathrm{C}-{ }^{13} \mathrm{C}-\mathrm{COSY}$ correlations. Off-diagonal responses denoted with solid red lines correspond to Type I artifact responses predicted by the icv-HSQC spectrum shown in Panel A. Off-diagonal responses designated with dashed red lines correspond to Type II responses. The blue boxes highlight the Type I artifact responses, contained in Panel B that are identified by the off-diagonal responses in the icv-HSQC spectrum shown in Panel A. The dashed blue box identifies the Type II response origin, which as shown in Figure 2, arise from the overlap of a direct response from one spin system and the relayed response from an overlapped, second spin system. 
calculate a icv-HSQC-COSY spectrum from the discrete gradient or non-gradient HSQC and COSY spectra has the potential to provide investigators with considerable time savings $v s$. having to acquire the hyphenated 2D data. In addition, this type of postacquisition processing also allows investigators access to GHSQC-COSY spectra when they might have to wait before they again have access to a spectrometer on which to acquire the data or worse still, when they may no longer have the sample due to decomposition, consumption for biological or other testing, etc.

Visual comparison of the unsymmetrical indirect covariance calculated icvHSQC-COSY and acquired IDR-GHSQC-TOCSY spectra shown in Figures 4B and 4A, respectively, quickly confirms that there are responses present in the icv-HSQC-COSY spectrum that are not present in the experimental spectrum. There are two possibilities for the additional responses. First, as expected, proton resonance overlaps confirmed by the icv-HSQC spectrum (Figures 1B and $3 \mathrm{~A}$ ) are expected to give rise to artifact responses. Second, there also exists the possibility that the higher relative sensitivity of the GHSQC and GCOSY experiments, when compared to the IDR-GHSQC-TOCSY experiment, can allow legitimate responses to be observed that are either very weak or even absent in the IDR-GHSQC-TOCSY spectrum.

Analyzing the icv-HSQC spectrum, ${ }^{13} \mathrm{C}$ chemical shift pairs can be determined for the off-diagonal response pairs. Plotting the GHSQC indirect covariance spectrum beside the icv-HSQC-COSY spectrum, the off-diagonal response pairs of the former correspond to ${ }^{13} \mathrm{C}$ shifts in the latter at which artifact responses may be observed as shown in Figure 4. Using the overlapped $\mathrm{H} 6$ and $\mathrm{H} 15$ proton resonances at $8.52 \mathrm{ppm}$ as an example, offdiagonal responses are observed in Figure 4C for C6 (125.4 ppm) and C15 (129.8 ppm) 

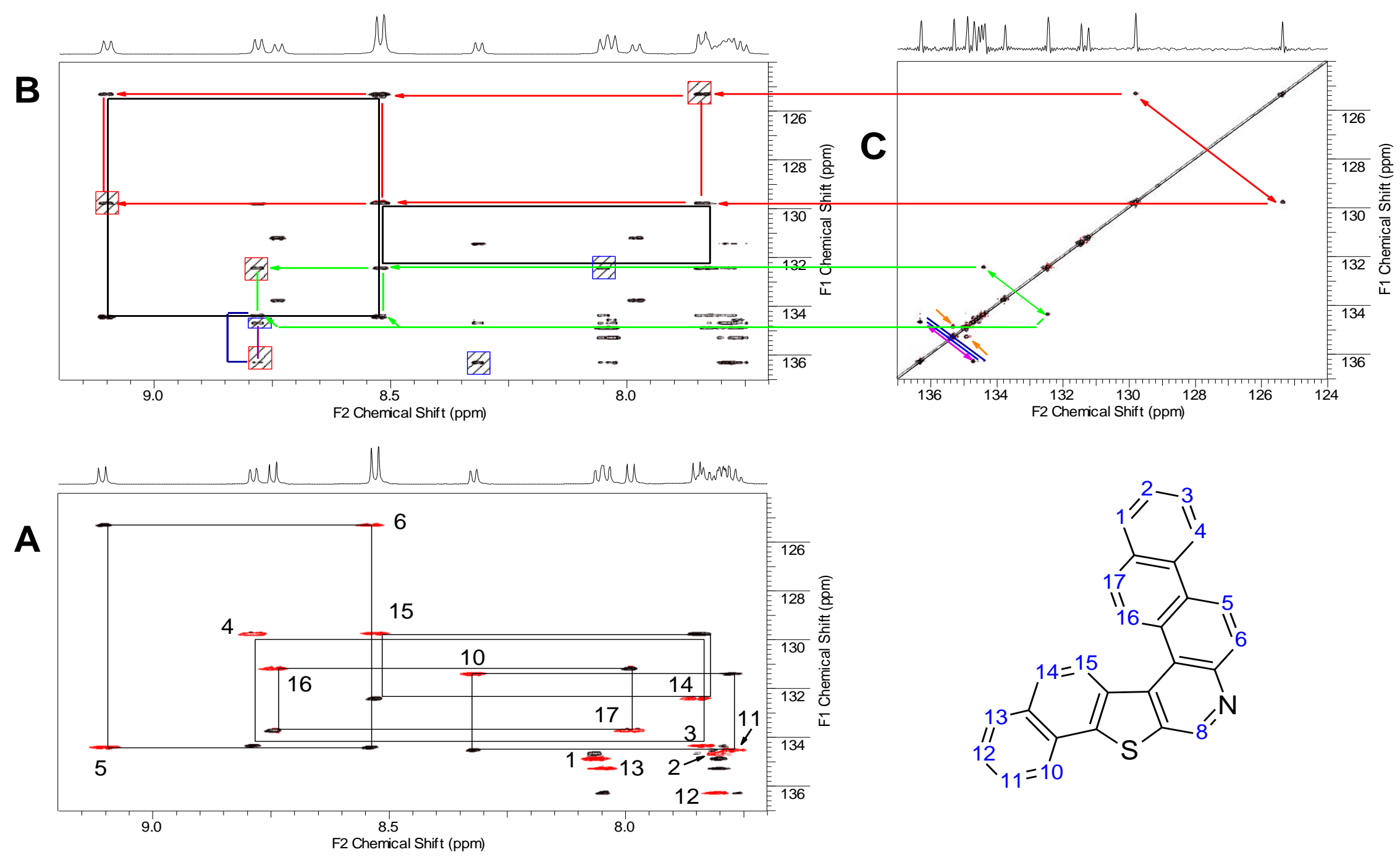

\section{Figure 4}


Figure 4. A.) IDR-GHSQC-TOCSY spectrum of 1 recorded at $600 \mathrm{MHz}$ with an $18 \mathrm{msec}$ mixing time. Direct responses are inverted and are plotted in red; TOCSY responses have positive intensity and are plotted in black. A high resolution proton spectrum is plotted above Panel A. Connectivities are shown in the less congested region of the spectrum. Direct proton/carbon correlation responses are labeled. ${ }^{10}$ B.) icv-HSQC-COSY spectrum calculated using the unsymmetrical indirect covariance processing algorithm from discretely acquired GHSQC and GCOSY spectra. A projection is shown above Panel B. C.) icv-HSQC spectrum of 1. A projected ${ }^{13} \mathrm{C}$ spectrum is shown above Panel C. Off-diagonal responses correspond to protonated carbons with overlapped protons. The $\mathrm{F}_{1}{ }^{13} \mathrm{C}$ shift corresponds to the $\mathrm{F}_{1}$ axis of the icv-HSQC-COSY spectrum shown in Panel B. The off-diagonal pair of responses in Panel C denoted with the red bar corresponds to the overlapped proton resonances associated with the C6 and C15 resonances at 125.4 and 129.8 ppm, respectively. Red arrows from the off-diagonal responses in Panel C to the corresponding $\mathrm{F}_{1}$ chemical shifts in Panel B denote the carbons associated with overlapped protons. Correlations at the proton chemical shifts of H14 (7.84 ppm), H6/H15 (8.52 ppm), and H5 (9.09 ppm) and the C6 (125.4 ppm) and C15 (129.8 ppm) correspond to artifact correlations. These pairs of responses in the icv-HSQC-COSY spectrum are designated by vertical red bars. Off-diagonal responses at 134.4 and 132.4 ppm in Panel C designated by the green bar correspond to another pair of ${ }^{13} \mathrm{C}$ shifts with proton resonance overlaps. Green arrows from Panel C to Panel B identify these pairs of artifact responses in a manner analogous to that just described from the H6/H15 proton resonances. Artifact correlations in the icv-HSQC-COSY spectrum shown in Panel B are identified by red hatched boxes. Open blue boxes designate legitimate correlations that are either not observed in the $18 \mathrm{msec}$ IDR-GHSQC-TOCSY spectrum shown in Panel A or responses that are below the threshold of the spectrum shown in Panel A. 
(denoted with red anti-diagonal line). Tracking horizontally from Figure 4C to Figure 4B, pairs of responses at these ${ }^{13} \mathrm{C}$ chemical shifts are observed at the proton shifts of H14 (7.84 ppm), H6/H15 (8.52 ppm), and H5 (9.09 ppm). The vertical red lines in Figure 4B denote the pairs of responses identified by the off-diagonal responses in Figure 4C. The responses in red, hatched boxes at 7.84/125.4 and 9.09/129.8 ppm are artifact responses. The responses at 8.52/125.4 and 8.52/129.8 ppm are both direct responses from isolated spin systems and hence are not correlated to one another. Off-diagonal responses at 134.4 and $132.4 \mathrm{ppm}$ in Panel C correlated with a green anti-diagonal line represent another pair of artifact correlations that are tracked back into Figure 4B. In a similar fashion, the other off-diagonal correlations shown in Figure 4C could be used to track artifacts in the highly congested region of the icv-HSQC-COSY spectrum in the region from 7.75-8.1 ppm in $\mathrm{F}_{2}$ and from 134.0-136.5 ppm in $\mathrm{F}_{1}$.

In addition to the artifact responses already discussed in the icv-HSQC-COSY spectrum shown in Figure 4B, there are also several additional responses in blue, hatched boxes. The responses in this group are legitimate correlation responses that are either below the threshold of the IDR-GHSQC-TOCSY contour plot shown in Figure 4A or not observed at all because of the length of the mixing time used. The ability to observe "distant" responses in a four-spin system is a function of the mixing time in the case of a GHSQC-TOCSY experiment or the extent of digitization in the $\mathrm{F}_{1}$ frequency domain in the case of the GCOSY spectrum used to calculate the icv-HSQC-COSY spectrum as shown in Figure 4B. 


\section{CONCLUSIONS}

Subjecting a GHSQC spectrum to indirect covariance processing gives rise to an auto-correlated icv-HSQC spectrum in which the only off-diagonal responses observed are due to carbon resonances with overlapped, directly attached protons as shown in Figure 1. As shown in Figure 3, the off-diagonal responses in the icv-HSQC spectrum can be used to identify artifact responses in GHSQC-TOCSY and IDR-GHSQC-TOCSY spectra subjected to indirect covariance processing to derive icv- ${ }^{13} \mathrm{C}-{ }^{13} \mathrm{C}-\mathrm{COSY}$ correlation plots. Perhaps the most valuable aspect of the present report is the demonstrated ability to use off-diagonal response information from the icv-GHSQC spectrum to identify artifacts in icv-HSQC-COSY spectra calculated from discretely acquired GHSQC and GCOSY spectra using the unsymmetrical indirect covariance processing algorithm.

Overlapping proton resonances, as initially observed in the late 2004 report of Zhang and Brüschweiler, ${ }^{1}$ can give rise to artifact responses in icv- ${ }^{13} \mathrm{C}-{ }^{13} \mathrm{C}-\mathrm{COSY}$ correlation plots calculated by indirect covariance processing of GHSQC-TOCSY and IDR-GHSQC-TOCSY spectra. In a similar fashion, proton resonance artifacts can also lead to artifacts in icv-HSQC-COSY spectra calculated using unsymmetrical indirect covariance processing as shown in the present study, or in icv- ${ }^{13} \mathrm{C}-{ }^{15} \mathrm{~N}$ HSQC-HMBC as recently demonstrated. ${ }^{10}$ However, as demonstrated in the present study, off-diagonal responses contained in the indirect covariance processed GHSQC can be used to "forecast" the location of artifact responses in indirect covariance and unsymmetrical 
indirect covariance calculated spectra of various types. Hopefully, the ability to determine the location of artifact responses in calculated icv-HSQC-COSY spectra will facilitate the use of these and other calculated spectra, affording investigators considerable spectrometer time savings. It will also be interesting to explore the application of icv-HSQC spectra in the examination of spectra derived by covariance processing of HMBC spectra as recently reported by Müller and co-workers. ${ }^{13}$

Some examples of time savings have been reported but as a further example, we wish to note that ${ }^{13} \mathrm{C}-{ }^{15} \mathrm{~N}$ heteronuclear correlation spectroscopy cannot be reasonably undertaken at natural abundance while, in contrast, these correlation spectra can be generated algorithmically. This capability alone further underscores the value of the unsymmetrical indirect covariance calculation of hyphenated and other 2D NMR spectra. 


\section{REFERENCES}

1. Zhang, F.; Brüschweiler, R. J. Am. Chem. Soc. 2004; 126: 13180.

2. Blinov, K. A.; Larin, N. I.; Kvasha, M. P.; Moser, A.; Williams, A. J.; Martin, G. E. Magn. Reson. Chem. 2005; 43: 999.

3. Blinov, K. A.; Larin, N. I.; Williams, A. J.; Zell, M.; Martin, G. E. Magn. Reson. Chem. 2006; 44, 107.

4. Blinov, K. A.; Larin, N. I.; Williams, A. J.; Mills, K. A.; Martin, G. E. J. Heterocycl. Chem. 2006; 43: 163.

5. Martin, G. E.; Hilton, B. D.; Irish, P. A.; Blinov, K. A.; Williams, A. J. J. Nat. Prod., 2007; in press.

6. Blinov, K. A.; Williams, A. J.; Hilton, B. D.; Irish, P. A.; Martin, G. E. Magn. Reson. Chem. 2007; 45: 544.

7. Martin, G. E.; Hilton, B. D.; Irish, P. A.; Blinov, K. A.; Williams, A. J. Magn. Reson. Chem. 2007; 45: 624.

8. Martin, G. E.; Hilton, B. D.; Irish, P. A.; Blinov, K. A.; Williams, A. J. J. Heterocycl. Chem. 2007; 44: 1219.

9. Martin, G. E.; Hilton, B. D.; Irish, P. A.; Blinov, K. A.; Williams, A. J. Magn. Reson. Chem., 2007; 45: 883.

10. Hadden, C. E.; Martin, G. E.; Luo, J.-K.; Castle, R. N. J. Heterocycl. Chem. 2000; 37: 821.

11. Martin, G. E.; Hilton, B. D.; Blinov, K. A.; Williams, A. J. J. Nat. Prod., 2007; submitted. 
12. For reviews of ASIS (Aromatic Solvent Induced Shifts) see the following: Laszlo, P. Prog. NMR Spectrosc., 1967; 3: pp. 231-402; Ronayne, J.;

Williams, D. H. Ann. Rev. NMR Spectrosc., 1969; 2: pp. 83-124.

13. Schoefberger, W.; Smrečki, V.; Vikić-Topić, D.; Müller, N. Magn. Reson. Chem., 2007; 45: 583. 